Modern Physics Letters B

(C) World Scientific Publishing Company

\title{
Classical thermodynamics from quasi-probabilities
}

\author{
F. Pennini ${ }^{1,2 *}$ \\ ${ }^{1}$ Facultad de Ciencias Exactas y Naturales, Universidad Nacional de La Pampa, Peru 151, \\ 6300 Santa Rosa, La Pampa, Argentina \\ ${ }^{2}$ Departamento de Física, Universidad Católica del Norte, Av. Angamos 0610, Antofagasta, \\ Chile \\ A. Plastino ${ }^{3}$ \\ ${ }^{3}$ Instituto de Física La Plata-CCT-CONICET, Universidad Nacional de La Plata, C.C. 727, \\ 1900, La Plata, Argentina \\ M.C. Rocca ${ }^{3}$ \\ ${ }^{3}$ Instituto de Física La Plata-CCT-CONICET, Universidad Nacional de La Plata, C.C. 727, \\ 1900, La Plata, Argentina \\ Received (Day Month Year) \\ Revised (Day Month Year)
}

\begin{abstract}
The basic idea of a microscopic understanding of Thermodynamics is to derive its main features from a microscopic probability distribution. In such a vein, we investigate the thermal statistics of quasi-probabilities's semi-classical analogs in phase space for the important case of quadratic Hamiltonians, focusing attention in the three more important instances, i.e., those of Wigner, $P_{-}$, and Husimi distributions. Introduction of an effective temperature permits one to obtain a unified thermodynamic description that encompasses and unifies the three different quasi-probability distributions. This unified description turns out to be classical.
\end{abstract}

Keywords: Semiclassical physics; $P$-function; Information quantifiers; thermal properties

\section{Introduction}

A quasi-probability (QP) distribution is a mathematical construction resembling a probability distribution. It does not necessarily fulfill, though, some of the Kolmogorov axioms for probabilities 1 . QPs display general features of ordinary probabilities and yield expectation values with respect to the weights of the distribution. However, they disobey the third probability postulate 1 , in the sense that regions integrated under them do not represent probabilities of mutually exclusive states. Some quasi-probability distributions exhibit zones of negative probability density.

${ }^{*}$ Corresponding author: flavia.pennini@gmail.com 
QPs often arise in trying to study quantum mechanics a phase space representation. This is frequently done in quantum optics, time-frequency analysis, etc.

The dynamics of a quantum system is determined by a master equation. We speak of an equation of motion for the density operator $(\hat{\rho})$, defined with respect to a complete orthonormal basis. One can show that the density can always be written in a diagonal manner, with respect to an overcomplete basis 2 . If this basis is that of coherent states $|\alpha\rangle$ one has $2 \mid 3$

$$
\hat{\rho}=\int \frac{d^{2} \alpha}{\pi} P\left(\alpha, \alpha^{*}\right)|\alpha\rangle\langle\alpha|
$$

where $\mathrm{d}^{2} \alpha / \pi=\mathrm{d} x \mathrm{~d} p / 2 \pi \hbar$, with $x$ and $p$ variables of the phase space. The system evolves as prescribed by the evolution of the quasi-probability distribution function. Coherent states, eigenstates of the annihilation operator $\hat{a}$, serve as the overcomplete basis in such a build-up 213 .

There exists a family of different representations, each connected to a different ordering of the creation and destruction operators $\hat{a}$ and $\hat{a}^{\dagger}$. Historically, the first of these is the Wigner quasi-probability distribution $W^{4}$, related to symmetric operator ordering. In quantum optics the particle number operator is naturally expressed in normal order and, in the pertinent scenario, the associated representation of the phase space distribution is the Glauber-Sudarshan $P$ one 3 . In addition to $W$ and $P$, one may find many other quasi-probability distributions emerging in alternative representations of the phase space distribution 5 . A quite popular representation is the Husimi $Q$ one $6 / 7 / 819$, used when operators are cast in anti-normal order.

In this paper we wish to apply semiclassical information theory tools associated to these $P, Q$, and $W$ representations (for quadratic Hamiltonians) in order to describe the concomitant thermal semiclassical features. We specialize things to the three $f$-functions associated to a Harmonic Oscillator (HO) of angular frequency $\omega$. In such a scenario the three functions -that we name for sake of convenience $f_{P}$, $f_{Q}$, and $f_{W^{-}}$are simple Gaussians and the treatment becomes entirely analytical, a very convenient feature. The $\mathrm{HO}$ is a really important system that yields insights usually having a wide impact. Thus, the HO constitutes much more than a mere simple example. Nowadays, it is of particular interest for the dynamics of bosonic or fermionic atoms contained in magnetic traps $10 / 1112$ as well as for any system that exhibits an equidistant level spacing in the vicinity of the ground state, like nuclei or Luttinger liquids.

In this paper we are interested in thermal states regarded as semi-classical distributions in phase space -analogs of the quantum quasi-probabilistic distributions. We will try to ascertain what physical Thermodynamics' features are they able to describe at the semi-classical level. These distributions are 1314

$$
f_{P}=\gamma_{P} e^{-\gamma_{P}|\alpha|^{2}}, \gamma_{P}=e^{\beta \hbar \omega}-1 \quad(P-\text { function })
$$




$$
\begin{gathered}
f_{Q}=\gamma_{Q} e^{-\gamma_{Q}|\alpha|^{2}}, \gamma_{Q}=1-e^{-\beta \hbar \omega} \quad(Q-\text { function }), \\
f_{W}=\gamma_{W} e^{-\gamma_{W}|\alpha|^{2}}, \gamma_{W}=2 \tanh (\beta \hbar \omega / 2) \quad(W-\text { function }),
\end{gathered}
$$

with $\beta=1 / k_{B} T, k_{B}$ the Boltzmann constant, and $T$ the temperature. As stated above, these distributions will be used in the next section as semiclassical statistical weight functions. Since ours is NOT a quantum approach, the ordering of the HO-creation and destruction operators $a$ and $a^{\dagger}$ plays no role whatsoever in our manipulations below.

This paper is organized as follows: section 2 refers to information quantifiers, in a phase space representation, for Gaussian distributions. In Section 3 we focus attention upon thermodynamic relations and we express them in terms of an effective temperature. Finally, some conclusions are drawn in Section 4.

\section{Semi-classical information quantifiers}

- The first step in our development is to calculate entropic quantifiers for these Gaussian distributions. In order to simplify the notation we will consider a general normalized gaussian distribution in phase space

$$
f(\alpha)=\gamma e^{-\gamma|\alpha|^{2}}
$$

whose normalized variance is $1 / \gamma$ and $\gamma$ taking values $\gamma_{P}, \gamma_{Q}$ and $\gamma_{W}$ given by Eqs. (2), (3), and (4), respectively.

The logarithmic Boltzmann's information measure for the the probability distribution (15) is

$$
S=-\int \frac{\mathrm{d}^{2} \alpha}{\pi} f(\alpha) \ln f(\alpha)=1-\ln \gamma,
$$

so that it acquires the particular values

$$
\begin{gathered}
S_{P}=1-\ln \left(e^{\beta \hbar \omega}-1\right), \\
S_{Q}=1-\ln \left(1-e^{-\beta \hbar \omega}\right), \\
S_{W}=1-\ln (2 \tanh (\beta \hbar \omega / 2)),
\end{gathered}
$$

for, respectively, the distributions $f_{P}, f_{Q}$, and $f_{W}$.

- Next, we focus attention on the information quantifier known as Fisher's information measure. We specialize it for families of shift-invariant distributions, that do not change shape under translations. One has 1516

$$
I=\int d x f(x)\left(\frac{\partial \ln f(x)}{\partial x}\right)^{2},
$$


and, in phase space, it adopts the appearance 17

$$
I=\frac{1}{4} \int \frac{\mathrm{d}^{2} \alpha}{\pi} f(\alpha)\left(\frac{\partial \ln f(\alpha)}{\partial|\alpha|}\right)^{2},
$$

such that considering $f(\alpha)$ given by Eq. (5) we get $I=\gamma$, whose specific values are $\gamma_{P}, \gamma_{Q}, \gamma_{W}$ for the three functions $f_{P}, f_{Q}$, and $f_{W}$, respectively.

- The statistical complexity is a functional $C[P]$ that can primarily be viewed as a quantity that characterizes the probability distribution $P$. It quantifies not only randomness but also the presence of correlational structures 18 . The opposite extremes of perfect order and maximal randomness possess no structure to speak of. In between these two special instances, a wide range of possible degrees of physical structure exist, degrees that should be reflected in the features of the underlying probability distribution. The statistical complexity $C$, according to López-Ruiz, Mancini, and Calvet 18 , is a suitable product of two quantifiers, such that $C$ becomes minimal at the extreme situations of perfect order or total randomness. Instead of using the prescription of 18 , but without violating its spirit, we will take one of these two quantifiers to be Fisher's measure and the other an entropic form, since it is well known that the two behave in opposite manner 19 . Thus:

$$
C=S I=\gamma(1-\ln \gamma)
$$

that vanishes for perfect order or total randomness. For each particular case, we explicitly have

$$
\begin{aligned}
C_{P} & =\left(e^{\beta \hbar \omega}-1\right)\left[1-\ln \left(e^{\beta \hbar \omega}-1\right)\right], \\
C_{Q} & =\left(1-e^{-\beta \hbar \omega}\right)\left[1-\ln \left(1-e^{-\beta \hbar \omega}\right)\right], \\
C_{W} & =(2 \tanh (\beta \hbar \omega / 2))[1-\ln (2 \tanh (\beta \hbar \omega / 2))],
\end{aligned}
$$

for, respectively, the distributions $f_{P}, f_{Q}$, and $f_{W}$. The maximum of the statistical complexity occurs when $\gamma=1$ and, the associated temperature values are

$$
\begin{cases}e^{\beta \hbar \omega}-1=1 \Rightarrow T=\hbar \omega / k_{B} \ln 2 & \text { for the } f_{P} \text {-function, } \\ 1-e^{-\beta \hbar \omega}=1 \Rightarrow T=0 & \text { for the } f_{Q} \text {-function, } \\ 2 \tanh (\beta \hbar \omega / 2)=1 \Rightarrow T=\hbar \omega / 2 k_{B} \arctan (1 / 2) & \text { for the } f_{W} \text {-function. }\end{cases}
$$




\section{Thermodynamic relations}

We start this section considering the semi-classical Hamiltonian of the harmonic oscillator that reads

$$
\mathcal{H}(x, p)=\hbar \omega|\alpha|^{2}=\hbar \omega\left(\frac{x^{2}}{4 \sigma_{x}^{2}}+\frac{p^{2}}{\sigma_{p}^{2}}\right)
$$

where $x$ and $p$ are phase space variables and $\sigma_{x}^{2}=\hbar / 2 m \omega$ and $\sigma_{p}^{2}=\hbar m \omega / 2 \underline{20}$. Let us further define the semiclassical expectation value of the function $\mathcal{A}(x, p)$ as

$$
\langle\mathcal{A}\rangle_{f}=\int \frac{\mathrm{d}^{2} \alpha}{\pi} f(\alpha) \mathcal{A}(x, p),
$$

indicating that $f(\alpha)$ is the statistical weight function. Thus, the mean energy of the hamiltonian $\mathcal{H}(x, p)$ is written in the fashion

$$
U^{*}=\hbar \omega \int \frac{\mathrm{d}^{2} \alpha}{\pi} f(\alpha)|\alpha|^{2}=\frac{\hbar \omega}{\gamma},
$$

where $\gamma$ takes the respective values $\gamma_{P}, \gamma_{Q}$, and $\gamma_{W}$ explained in Introduction. Additionally, the thermodynamic entropy $S^{\prime}$ is

$$
S^{\prime}=k_{B}(1-\ln \gamma)
$$

where we have added the Boltzmann constant $k_{B}$. The mean energy can be viewed as a function of the thermodynamic entropy $S^{\prime}$, in the following fashion. Combining (19) with the thermodynamic entropy (20) we get the associated, fundamental equation $U^{*}=U^{*}\left(S^{\prime}\right)$

$$
U^{*}\left(S^{\prime}\right)=\hbar \omega e^{S^{\prime} / k_{B}-1},
$$

and

$$
\gamma=e^{1-S^{\prime} / k_{B}}
$$

Thus, the differential of $U^{*}$ becomes

$$
\mathrm{d} U^{*}=\left(\frac{\partial U^{*}}{\partial S^{\prime}}\right)_{V} \mathrm{~d} S^{\prime},
$$

where we have considered the volume $V$ to be constant. Thus, after effecting the pertinent replacements we find

$$
\mathrm{d} U^{*}=\frac{\hbar \omega}{k_{B} \gamma} \mathrm{d} S^{\prime},
$$

which suggests introducing an effective temperature $T_{\text {eff }}$. Using $T_{\text {eff }}$ we obtain a unified picture that encompasses the three distributions $f_{P}, f_{Q}$, and $f_{W}$, in a single thermodynamic description. We have

$$
T_{e f f}=\left(\frac{\partial U^{*}}{\partial S^{\prime}}\right)_{V}=\frac{\hbar \omega}{k_{B} \gamma}
$$


such that

$$
\mathrm{d} U^{*}=T_{e f f} \mathrm{~d} S^{\prime} .
$$

Note that in the three instances, $T_{\text {eff }}=\infty$ for $T=\infty$. However, if $T=0$, $T_{\text {eff }}=0$ only in the $f_{P}$-case. It equals $1 / 2$ in the Wigner instance and equals 1 in the Husimi case, as depicted in the accompanying figure.

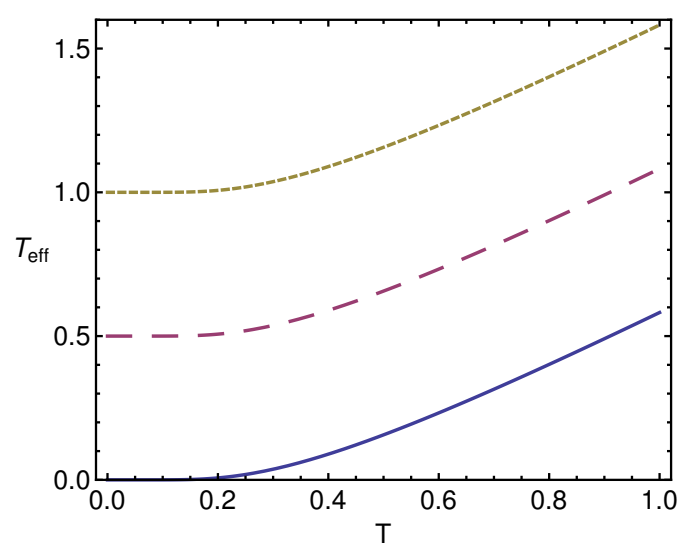

Fig. 1. Effective temperature $T_{\text {eff }}$ versus temperature $T$ in $\left(\hbar \omega / k_{B}\right)-$ units.

From (19) and (26) we can rewrite the mean energy in terms of effective temperature.

$$
U^{*}=k_{B} T_{e f f},
$$

that corresponds to the classical mean energy of a harmonic oscillator of temperature $T_{\text {eff }}$, with $k_{B} T_{\text {eff }} / 2$ contributions for each of the two pertinent degrees of freedom. Similarly, the thermodynamic entropy is recast as

$$
\frac{S^{\prime}}{k_{B}}=1+\ln \left(\frac{k_{B} T_{e f f}}{\hbar \omega}\right)
$$

and the Helmholtz free energy is given by

$$
A^{*}=U^{*}-T_{e f f} S^{\prime}=k_{B} T_{e f f} \ln \left(\frac{\hbar \omega}{k_{B} T_{e f f}}\right) .
$$

The effective specific heat is defined as

$$
C_{V}^{*}=\left(\frac{\partial U^{*}}{\partial T_{e f f}}\right)_{V}
$$

that using (27) becomes

$$
C_{V}^{*}=k_{B}
$$


which is precisely the specific heat for the classical harmonic oscillator which is independent of the temperature. This becomes the Dulong and Petit's rule at the classical limit. In view of (26) and (29) the analog partition function $Z^{*}$ is given by

$$
Z^{*}=\frac{1}{\gamma}
$$

and, according to Eqs. (6), (19), and (32) we find

$$
S^{\prime}=\ln Z^{*}+\beta^{*} U^{*},
$$

with

$$
\beta^{*}=\frac{1}{k_{B} T_{e f f}}=\frac{\gamma}{\hbar \omega} \text {. }
$$

Thus, one reobtains all the thermal results pertaining to a classical $\mathrm{HO}$ at the temperature $T_{\text {eff }}$. Note that the whole thermal description becomes now of a classical character. All the quantum effects pertaining to the probability distributions are contained in the relationship (24) between $T_{\text {eff }}$ and $T$.

It is interesting to look at the statistical complexity $C$ in order to see at what effective temperature the possible correlational structures carried by our probabilistic distributions are stronger. Expressed in terms of $T_{\text {eff }}, C$ becomes

$$
C^{\prime}=I S^{\prime}=\frac{\hbar \omega}{T_{e f f}}\left[1+\ln \left(\frac{k_{B} T_{e f f}}{\hbar \omega}\right)\right] .
$$

Keeping in mind $T_{e f f}$ 's definition, it is easy to see that the maximum for the complexity $C^{\prime}$ is attained when $T_{e f f}=\hbar \omega / k_{B}$. This implies, according to Eq. (25) that the maximum of the Fisher measure es $I_{\max }=1$. At the complexity-peak, thermodynamic quantities take the values

$$
\begin{aligned}
& U_{\text {max }}^{*}=\hbar \omega, \\
& S_{\text {max }}^{\prime}=k_{B}, \\
& C_{\text {max }}^{\prime}=k_{B},
\end{aligned}
$$

a remarkable simplicity!

\section{Conclusions}

We have investigated here the thermal statistics of quasi-probabilities-analogs $f(\alpha)$ in phase space for the important case of quadratic Hamiltonians, focusing attention on the three more important instances, i.e., those of Wigner, $P_{-}$, and Husimi distributions. 
- Introduction of an effective temperature permits one to obtain a unified thermodynamic description that encompasses the three different quasiprobability distributions. This unified description turns out to be classical.

- The above entails that all possible "quasi-quantum" effects have to be contained in the relationship between $T_{\text {eff }}$ and $T$. Note, for instance, that the minimal energy is not zero (one of these effects) but, in the $f_{W}$-case, $T_{\text {eff }}^{W}=\hbar \omega / 2 k_{B}$, implying a minimum energy $k_{B} T_{\text {eff }}^{W}=\hbar \omega / 2$. Additionally, the Husimi- $T_{e f f}^{Q}=\hbar \omega / k_{B}$ reflects the well known fact that the Husimi distribution "smoothes" the Wigner one over a phase-space area $=\hbar$.

- The basic idea of a microscopic understanding of thermodynamics is to derive its main features from a microscopic probability distribution. We have done just this using as distributions quasi-probability ones.

\section{References}

1. J. Von Plato, Grundbegriffe der Wahrscheinlichkeitsrechnung in Grattan-Guinness, I., ed., (Landmark Writings in Western Mathematics, Elsevier, Amsterdam, pp 960-69, 2005).

2. E.C.G. Sudarshan, Physical Review Letters 10 (1963) 277.

3. R.J. Glauber, Physical Review 131 (1963) 2766.

4. E.P. Wigner, Physical Review 40 (1932) 749.

5. F. Pennini, A. Plastino, The European Physical Journal D 61 (2011) 241.

6. K. Husimi, Proceedings of the Physico-Mathematical Society of Japan 22 (1940) 264.

7. S.S. Mizrahi, Physica A 127 (1984) 241.

8. S.S. Mizrahi, Physica A 135 (1986) 237.

9. S.S. Mizrahi, Physica A 150 (1988) 541.

10. M.H. Anderson, J.R. Ensher, M.R. Matthews, C.E. Wieman, E.A. Cornell, Science 269 (1995) 198.

11. K.B. Davis, M.O. Mewes, M.R. Andrews, N.J. van Druten, D.S. Durfee, D.M. Kurn, W. Ketterle, Physical Review Letters 75 (1995) 3969.

12. C.C. Bradley, C.A. Sackett, R.G. Hulet, Physical Review Letters 78 (1997) 985.

13. M.O. Scully, M.S. Zubairy, Quantum optics, (Cambridge University Press, NY, 1997).

14. W.P. Scheleich, Quantum Optics in Phase Space, (Wiley VCH-Verlag, Berlin, Germany, 2001).

15. B.R. Frieden, B.H. Soffer, Physical Review E 52 (1995) 2274.

16. M.J.W. Hall, Physical Review A 62 (2000) 012107.

17. F. Pennini, A. Plastino, Entropy 16 (2014) 1178.

18. R. López-Ruiz, H.L. Mancini, X.A. Calbet, Phys. Lett. A 209 (1995) 321.

19. B.R. Frieden, Science from Fisher Information, 2 ed., (Cambridge University Press, Cambridge, UK, 2008).

20. R.K. Pathria, Statistical Mechanics, (Pergamon Press, Exeter, 1993). 


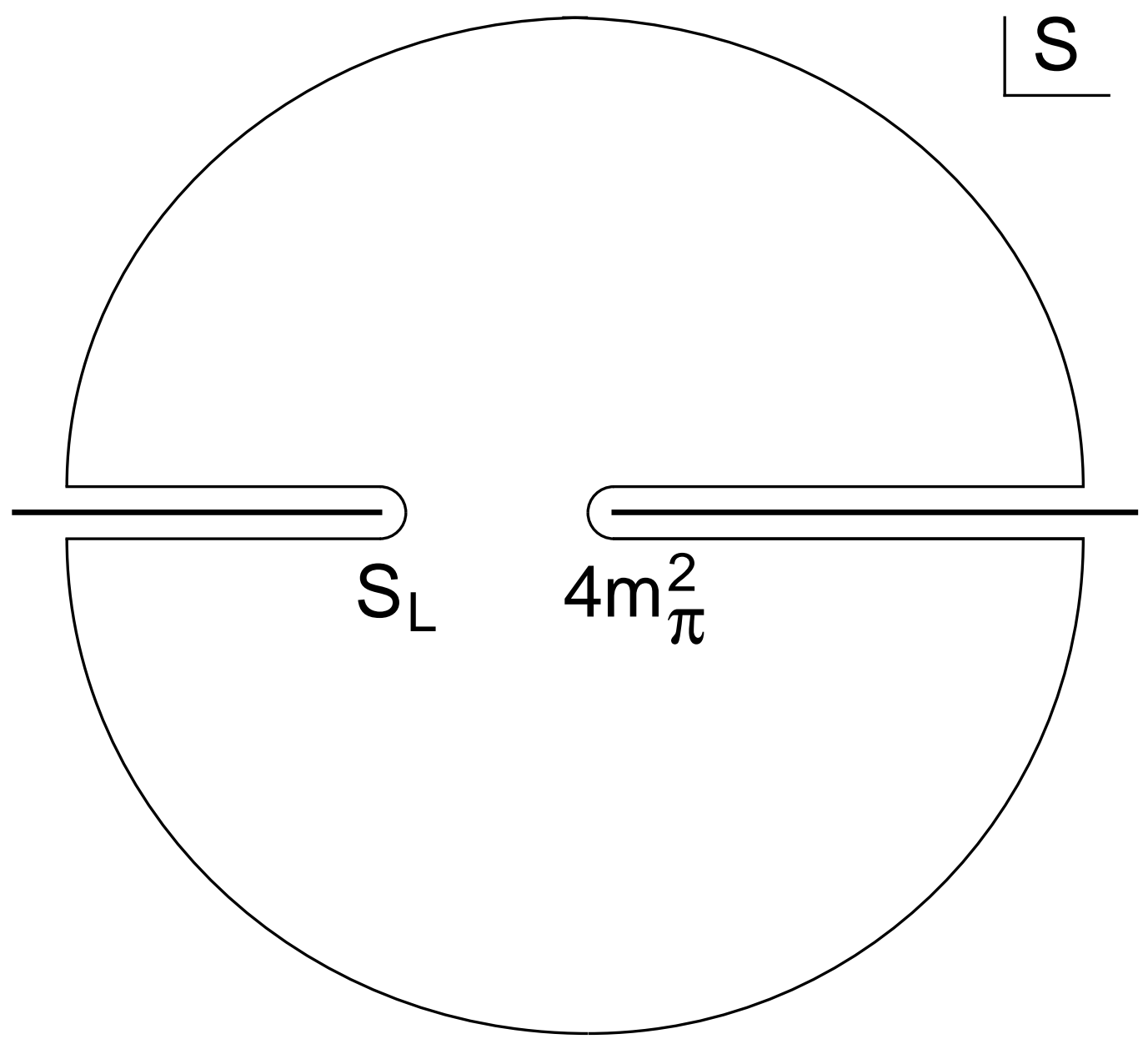

